\title{
Big Bang-Big Crunch Algorithm for Dynamic Deployment of Wireless Sensor Network
}

\author{
R.S. Uppal*, Shakti Kumar** \\ * Department of Computer Science and Engineering, BBSBEC Fatehgarh Sahib \\ ** Baddi University of Emerging Sciences \& Technology Baddi
}

\section{Article Info \\ Article history: \\ Received Jul 1, 2015 \\ Revised Nov 25, 2015 \\ Accepted Dec 16, 2015 \\ Keyword: \\ Abc algorithm \\ Big bang big crunch \\ Dynamic deployment \\ Sensor coverage}

\section{Corresponding Author:}

R S Uppal,

Department of Computer Science and Engineering,

Baba Banda Singh Bahadur Engineering College,

Fatehgarh Sahib, Punjab India 140407.

Email: rsuppal@gmail.com

\begin{abstract}
This paper proposes soft computing technique Big Bang-Big Crunch (BB$\mathrm{BC})$ to address the main issue of deployment of wireless sensor networks. Deployment is the main factor that significantly affects the performance of the wireless sensor network. This approach maximizes the coverage area of the given set of sensors. We implemented our approach in MATLAB and compared it with $\mathrm{ABC}$ approach and found that the proposed approach is much better than the said approach.
\end{abstract}

Copyright (c) 2016 Institute of Advanced Engineering and Science. All rights reserved.

\section{INTRODUCTION}

Emergence of Wireless Sensor Networks in the recent era has revolutionize the monitoring and surveillance activities in the field of communication. The study of Wireless Sensor Networks requires vast breadth of knowledge from variety of disciplines [1]. Wireless Sensor Network is understood as a collection of nodes organized into a cooperative network [10 from 2]. These inexpensive, low power communication nodes can be deployed throughout a physical space, providing dense sensing close to physical phenomena, processing and communicating the information, and coordinating actions with other nodes [3]. Wireless Sensor Network consists of thousands sensor nodes, deployed either randomly or according to some predefined statistical distribution, over geographic region of interest [5]. Wireless Sensor Networks find diversified application ranging from monitoring biological system to monitoring forest fires through air dropped sensors. Depending upon the area of interest, the placement of the sensors may be located at predetermined location while other placement could be optimally determined using computational intelligence [4]. Sensing and communication are two primary functions of Wireless Sensor Networks. The effectiveness of sensing and communication is determined by coverage and connectivity of the network. Coverage and connectivity issues largely depend upon the efficient deployment of the nodes in the interest region. Conversely, deployment decisions require optimal calculations of the network's coverage ratio, while maintaining the desired connectivity [4], [7]. One important criterion for being able to deploy an efficient sensor network is to find optimal node placement strategies. Deploying nodes in large sensing fields requires efficient topology control [6]. Due to the diversity of applications, requirements and design goals no single distinctive approach is available to the design and deployment of sensors networks [8]. The major issue in the deployment is to find the optimal placement of nodes, so that a minimum number of them are needed [9]. 
Optimal placement of nodes means finding the optimal location of sensors in the region of interest of deployment. This is a solution of Mathematical Problem having an objective function to be maximized or minimized within constraints. In the ROI, coverage ratios of different placements are computed and selection of optimal placements could be done by various optimization techniques [4].

Locations of Wireless Sensor Nodes computed in optimal solution of the technique would solve deployment issue of WSN. The deployment position of node is considered by its location coordinate (X, Y). To find out the position of these location coordinates while maintain connectivity and sensing in the target region is the key issue of deployment. The optimal location coordinates of wireless sensor nodes are to be computed using various algorithms for the optimal deployment of wireless sensor network. The region of interest is a two-dimensional grid and the initial deployment of nodes for algorithm is chosen randomly considering random location coordinates.

There are various algorithms which could be used to compute locations of nodes and coverage. The most recent algorithm is Artificial Bee Colony (ABC) algorithm which provides benefit of numerical optimization and clustering. The coverage problem is also optimized using Particle Swan Optimization (PSO) to give the best coverage by computing the location of sensor nodes placement [10].

In this study, we have proposed a new approach of Big Bang Big Crunch algorithm for dynamic deployment of sensors. We have also compared this algorithm to $\mathrm{ABC}$ algorithm which shows better deployment.

\section{BIG BANG-BIG CRUNCH ALGORITHM}

This is nature inspired optimization technique based on theory of Big Bang theory of universe. In Big Bang phase sensor placement is selected randomly and then in Big Crunch phase minimizes fitness function thereby giving optimal deployment and coverage.

Begin

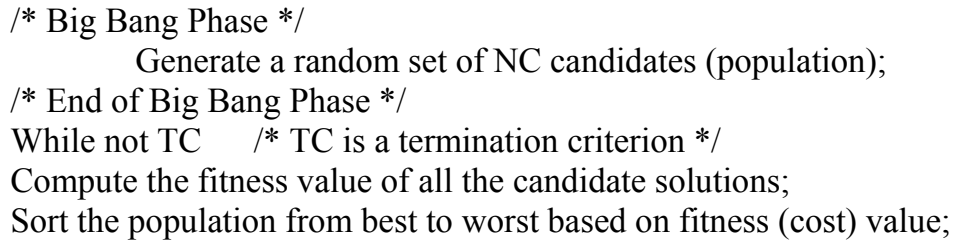

For guiding the new search compute the center of mass using following Equation;

$$
x_{c}=\frac{\sum_{i=1}^{N C} f^{i} x_{i}}{\sum_{i=1}^{N C} \frac{1}{f^{i}}}
$$

Where $\mathrm{x}_{\mathrm{c}}=$ position of the center of mass; $\mathrm{x}_{\mathrm{i}}=$ position of candidate $\mathrm{i} ; \mathrm{f}=$ fitness function value of candidate i;

Best fit individual can be chosen as the center of mass instead of using Eq.1;

/* End of Big Crunch Phase */

Calculate new candidates around the center of mass by adding or subtracting a normal random number hose value decreases as the iterations elapse using Equation;

$$
x^{n e w}=x^{c}+l(\text { rand }) / k
$$

Where $\mathrm{x}_{\mathrm{c}}$ stands for center of mass, $l$ is the upper limit of the parameter, rand is a normal random number and $\mathrm{k}$ is the $\mathrm{k}^{\text {th }}$ iteration of the algorithm.

Then new point $\mathrm{x}^{\text {new }}$ is upper and lower bounded. End while

End 


\section{SENSOR DETECTION MODEL}

Sensor detection models can be categorized in two ways in WSNs to find out the effective coverage area. One of the models is based on binary detection which is based on assumption that there is no uncertainty and the other model is based on probabilistic detection model [12] which provides more realistic results as compare to first one because it uses probabilistic terms for deciding the effective coverage of the area [6].

The binary detection model is adopted here [11].

Coverage ratio of the WSN is calculated using the Equation 1:

$$
C R=\frac{\cup c_{i}}{A}, i \in S
$$

Where, $C_{i}$ denotes the coverage area of a sensor i, S denotes the set of the nodes, and A is the total area of interest.

In this model, for a two-dimensional physical space, each sensor node range is considered as circle and is placed at the centre say (x, y) where $\mathrm{x}$ and $\mathrm{y}$ are coordinates of centre. In area A, $\mathrm{n}$ set of mobile nodes such as $\mathrm{S}=\left\{\mathrm{S}_{1}, \mathrm{~S}_{2}, \ldots \ldots \ldots \ldots, \mathrm{S}_{\mathrm{n}}\right\}$ is to be deployed at $\mathrm{n}$ different location having coordinates $\left(\mathrm{x}_{\mathrm{i}}, \mathrm{y}_{\mathrm{i}}\right)$, so as to ensure optimal coverage. The radius $r$ of the circle is the sensing range of sensor. Thus, the sensing area is $\pi r^{2}$, while its communication range is equal and greater than twice the sensing range. So, in a given twodimensional physical space of area $\mathrm{A}$, the $\mathrm{n}$ numbers of sensors are randomly deployed. The coordinates of centre of circles are denoted as $\left(x_{i}, y_{i}\right)$, where $i=1,2,3$, ..., $n$

The area of overlapping between sensing of two sensors $i$ and $j$ is represented by $A_{i j}$ and is calculated by

$$
A_{i j}=2 r^{2} \cos ^{-1}\left(\frac{d_{\bar{g}}}{2 r}\right)-\left(\frac{d_{i j}}{2}\right)\left(\sqrt{4 r^{2}-\left(G_{i i^{2}}\right)}\right)
$$

Where,

$\mathrm{d}_{\mathrm{ij}}$ is the distance between the sensor $\mathrm{i}$ and sensor $\mathrm{j}$ and is calculated by

$$
\begin{aligned}
& d_{i j}=\sqrt{\left(x_{j}-x_{i}\right)+\left(y_{j}-y_{i}\right)^{p}} \\
& \text { Total Overlapping of Deployment }=\frac{1}{2} \sum_{i}^{n} \sum_{i}^{n} A_{i}
\end{aligned}
$$

The area of overlapping of every sensor with the other (n-1) sensor is represented in the form of square matrix of order $n$ called the Area Matrix.

\section{SIMULATION RESULTS AND ANALYSIS}

We implemented the proposed deployment of BBBC algorithm using MATLAB. In an area of 100 X 100 square meter, 80 mobile sensors were deployed. Detection radius of each sensor was 7 meter and population size was 20 . 


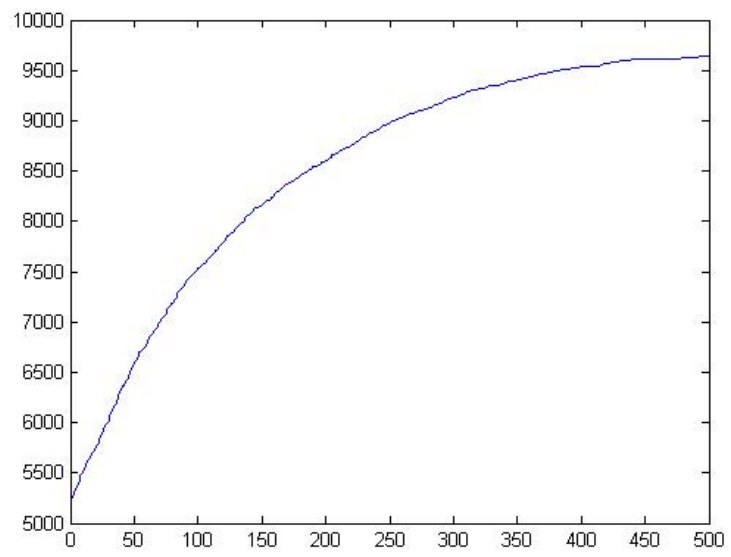

Figure 1. Coverage Area of the deployment vs 500 iterations

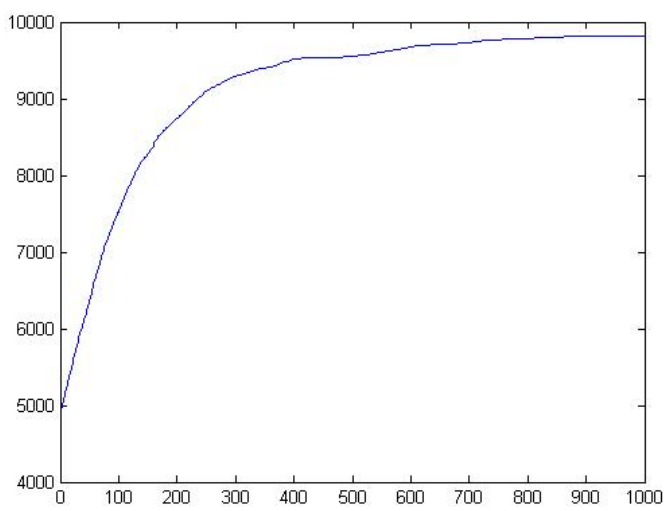

Figure 2. Coverage Area of the deployment vs 1000 iterations

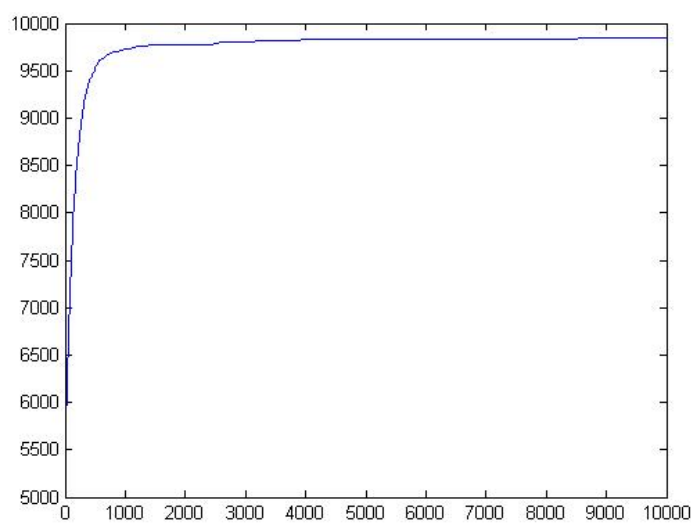

Figure 3. Coverage Area of the deployment vs 10000 iterations

BBBC algorithm was run with 500, 1000 and 10,000 iterations. We compared the performance of our proposed deployment approach with ABC approach in Table 1. 
Table 1. Comparison of $\mathrm{ABC}$ and $\mathrm{BB}-\mathrm{BC}$ Approach

\begin{tabular}{|c|c|c|c|c|}
\hline \multirow[t]{2}{*}{ Approach } & \multirow[t]{2}{*}{ No of Sensors } & \multicolumn{3}{|c|}{ No. of Iterations } \\
\hline & & 500 & 1000 & 10000 \\
\hline$\overline{\mathrm{ABC} \text { [27] }}$ & 100 & $96.66 \%$ & $98.33 \%$ & $99.34 \%$ \\
\hline BB-BC & 80 & $96.98 \%$ & $98.60 \%$ & $99.52 \%$ \\
\hline
\end{tabular}

\section{CONCLUSION}

In this paper, we have proposed BB-BC Approach for the deployment of mobile wireless sensors. The approach was implemented in MATLAB with an initial population of size 20, detection radius 7 and with three different sizes of iterations, viz. 500, 1000 and 10000. It was observed that in all the three iteration procedures, the proposed approach with 80 sensors performed better than the ABC approach with 100 sensors. The coverage area was improved upon by using the BB-BC approach even with lesser number of sensors. Thus, it is concluded that $\mathrm{BB}-\mathrm{BC}$ is a better deployment technique for mobile sensors than the $\mathrm{ABC}$ approach.

\section{REFERENCES}

[1] L. Lewis, "Wireless sensor networks in Smart Environments: Technologies, Protocols, and Applications", D.J. Cook and S.K. Das, Eds., chapter 4, John Wiley \& Sons, New York, NY, USA, 2004.

[2] Stankovic John A., "Wireless Sensor Networks", University of Virginia Charlottesville, Virginia 22904, June 19, 2006.

[3] David Culler, Deborah Estrin, and Mani Srivastava, Guest Editors', "Introduction: Overview of Sensor Networks", IEEE Computer, Vol. 37, No. 8, August 2004.

[4] Andrew Howard, Maja J Matarić, and Gaurav S. Sukhatme, "An Incremental Self-Deployment Algorithm for Mobile Sensor Networks", Autonomous Robots, special issue on Intelligent Embedded Systems, G. Sukhatme, ed., 2002, 113-126.

[5] Ghosh, Amitabha; Das, Sajal K., "Coverage and Connectivity Issues in Wireless Sensor Networks, Mobile, Wireless, and Sensor Networks: Technology, Applications, and Future Directions”, John Wiley \& Sons, Inc., 2006, pp. 221-256.

[6] Paolo Santi, "Topology Control in Wireless Ad Hoc and Sensor Networks", ACM computing surveys (CSUR), Vol. 37, No. 2, pp. 164-194, June 2005.

[7] Corke P, Hrabar S, Peterson R, Rus D, Saripalli S, Sukhatme G., “Autonomous Deployment and Repair of a Sensor Network using an Unmanned Aerial Vehicle", IEEE International Conference on Robotics and Automation (ICRA) 2004.

[8] Jan Beutel, Kay Romer, Matthias Ringwald, and Matthias Woehrle "Deployment Techniques for Sensor Networks" Springer in Signals and Communication Technology", 2009, Sensor Networks, Part 3, pp. 219-248.

[9] S Toumpis, L Tassiulas., "Information Theory", IEEE Transactions, Vol. 52, No. 7, pp. 2935-2953, 2006.

[10] N.A.B.A. Aziz and A.W. Mohemmed and M.Y. Alias, "A wireless sensor network coverage optimization algorithm based on particle swarm optimization and Voronoi diagram", International Conference on Networking, Sensing and Control, ICNSC, pp. 602-607, March 2009.

[11] X. Wang, S. Wang, and J. Ma, “An Improved Co-Evolutionary Particle Swarm Optimization for Wireless Sensor Networks with Dynamic Deployment”, Sensors, Vol. 7, No. 3, pp. 354-370, March 2007.

[12] Zou Yi, Chakrabarty Krishnendu, "Sensor Deployment and Target Localization in Distributed Sensor Networks ACM Transactions on Embedded Computing Systems 2003, Vol. 2, No. 3, pp. 1-29, 2003.

[13] Joseph Polastre, Robert Szewczyk, Alan Mainwaring, David Culler, John Anderson, "Analysis of Wireless Sensor Networks for Habitat Monitoring", Wirelss Sensor Networks, pp. 399-423, 2009.

[14] Ossama Younis, Marwan Krunz, and Srinivasan Ramasubramanian, "Node Clustering in Wireless Sensor Networks: Recent Developments and Deployment Challenges", IEEE, Vol. 20, No. 3, pp. 20-25, 2006.

[15] P. Gajbhiye, A. Mahajan, "A survey of architecture and node deployment in Wireless Sensor Network", First International Conference on the Applications of Digital Information and Web Technologies (ICADIWT 2008), pp. 426-430, 2008.

[16] Pompili, D.; Melodia, T. \& Akyildiz, I.F., "Deployment analysis in underwater acoustic wireless sensor networks", Underwater Networks, ACM, pp. 48-55.

[17] Xue Wang, Sheng Wang and Jun-Jie Ma, “An Improved Co-evolutionary Particle Swarm Optimization for Wireless Sensor Networks with Dynamic Deployment”, Vol. 7, No. 3, pp. 354-370, 2007.

[18] Honghai Zhang, Jennifer C. Hou, "Is Deterministic Deployment Worse than Random Deployment for Wireless Sensor Networks", IEEE, pp. 1-13, 2006.

[19] Nojeong Heo and Pramod K. Varshney, "Energy-Efficient Deployment of Intelligent Mobile Sensor Networks", IEEE, Vol. 35, No.1, pp. 78-92, 2005.

[20] Stavros Toumpis, Leandros Tassiulas, "Optimal Deployment of Large Wireless Sensor Networks”, IEEE, Vol. 52, No. 7, pp. 2953-2953, 2006.

[21] Shuhui Yang, Minglu Li, and Jie Wu, "Scan based Movement-Assisted Sensor Deployment Methods in Wireless Sensor Networks", Vol. 18 No. 8, pp. 1108-1121, 2007. 
[22] X. Liu and P. Mohapatra, "On the deployment of wireless sensor nodes", Third International Workshop on Measurement, Modelling, and Performance Analysis of Wireless Sensor Networks, 2005.

[23] Peng Cheng, and Chen-Nee Chuah Xin Liu, "Energy-aware Node Placement in Wireless Sensor Networks", Vol. 5, pp. $3210-3214$.

[24] Koushik Kar, Suman Banerjee, "Node Placement for Connected Coverage in Sensor Networks", Sophia-Ant polis, France, 2003.

[25] Md. Obaidur Rahman, Md. Abdur Razzaque and Choong Seon Hong, "Probabilistic Sensor Deployment in Wireless Sensor Network: A New Approach", Proceedings of the $9^{\text {th }}$ IEEE ICACT 2007, Vol. 2, pp. 1419-1422, Feb. 2007.

[26] Nojeong Heo and Pramod K. Varshney, "An Intelligent Deployment and Clustering Algorithm for a Distributed Mobile Sensor Network," Proceedings of the 2003 IEEE International Conference on Systems, Man \& Cybernetics, Vol. 5, pp. 4576-4581, Oct. 2003.

[27] Ozturk, C; Karaboga, D; Gorkemli, B. Artificial Bee Colony Algorithm for Dynamic Deployment of Wireless Sensor Networks. Turk J Elec Engi Compu Sci. Vol. 20, No. 2, 2012, pp. 255-262.

[28] Erol O.K., Eksin I., A new optimization method: Big Bang-Big Crunch, Advances in Engineering Software, vol 37, 2006, pp. 106-111.

[29] Shakti Kumar, Sukhbir Singh Walia \& A Kalanidhi, Fuzzy model identification: a new parallel BB-BC optimization based approach, International Journal of Electronics and Communication Engineering (IJECE) ISSN(P): 2278-9901; ISSN(E): 2278-991X Vol. 2, Issue 5, Nov 2013, pp. 167-178.

[30] Shakti Kumar, Sukhbir Singh Walia, Amar Singh, Parallel Big Bang-Big Crunch Algorithm, International Journal of Advanced Computing, Vol.46, Issue.3 1, Sept 2013. 